\title{
EULERIAN INTEGRAL FORMULAS AND ASSOCIATED FRACTIONAL INTEGRATION
}

\author{
MRIDULA GARG AND MRIDULA PUROHIT
}

\begin{abstract}
In the present paper, we evaluate two unified Eulerian integrals whose integrands involve the product of a general class of multivariable polynomials and the multivariable $\mathbf{H}$ function. The results obtained here provide interesting unifications and extensions of several known [eg. [9], [11], [12], [18], [21], etc.] and new results. We have given two new special cases which are also sufficiently general in nature and of interest in themselves. As an application, one of the result has also been expressed as fractional integral which would provide useful generalization of known results in the theory of fractional calculus.
\end{abstract}

\section{Introduction}

A large number of Eulerian integrals involving $\mathbf{H}$-function of one or more variables have been established by a number of research workers such as Srivastava and Singh [21], Gupta and Soni [6], Raina and Srivastava [8], Saxena and Nishimoto [11], Srivastava and Hussain [18], Saigo and Raina [9], Garg and Gupta [5] and many others. In the present paper we shall obtain two main Eulerian integrals which provide generalizations of some of the results mentioned above. The integrals obtained here involve product of a general class of multivariable polynomial and the multivariable $\mathbf{H}$-function.

Several generalizations of the multivariable polynomials have been studied by different authors from time to time namely Carlitz and Srivastava [2], Carlitz [1], Chak [3], Erdelyi [4], etc. [see also [21, Ch. 9]]. However, we shall consider here the following two general class of multivariable polynomials

(i) The first class of multivariable polynomials introduced by Srivastava and Garg $[16$, p. 686 , eq. (1.4)] as follows

$$
\begin{array}{r}
S_{V}^{U_{1}, \ldots, U_{r}}\left[x_{1}, \ldots, x_{r}\right]=\sum_{k_{1}, \ldots, k_{r}=0}^{U_{1} k_{1}+\cdots+U_{1} k_{r} \leq V}(-V) U_{1} k_{1}+\cdots+U_{r} k_{r} A\left(V ; k_{1}, \ldots, k_{r}\right) \frac{x_{1}^{k_{1}}}{k_{1} !} \cdots \frac{x_{r}^{k_{r}}}{k_{r} !} \\
V=0,1,2, \ldots
\end{array}
$$

Received June 9, 1999.

2000 Mathematics Subject Classification. 33A35, 26A33.

Key words and phrases. Eulerian integral, fractional integration, multivariable polynomials, multivariable $\mathbf{H}$-function. 
where $U_{1} \cdots U_{r}$ are arbitrary positive integers and the coefficient $A\left(V ; k_{1}, \ldots, k_{r}\right)$ are arbitrary constants, real or complex.

(ii) The second general class of multivariable polynomials given by Srivastava [14] defined and represented in the following slightly modified from

$$
\begin{aligned}
S_{V_{1}, \ldots, V_{r}}^{U_{1}, \ldots, U_{r}}\left[x_{1}, \ldots, x_{r}\right]= & \sum_{k_{1}=0}^{\left[V_{1} / U_{1}\right]} \cdots \sum_{k_{r}=0}^{\left[V_{r} / V_{r}\right]}\left(-V_{1}\right) U_{1} k_{1} \cdots\left(-V_{r}\right) U_{r} k_{r} \\
& \times A\left(V_{1}, k_{1} ; \ldots ; V_{r}, k_{r}\right) \frac{x_{1}^{k_{1}}}{k_{1} !} \cdots \frac{x_{r}^{k_{r}}}{k_{r} !}
\end{aligned}
$$

where $V_{i}=0,1,2, \ldots[i=1, \ldots r], U_{1}, \ldots, U_{r}$ are arbritrary positive integers, the coefficients $A\left[V_{1}, k_{1} ; \ldots ; V_{r}, k_{r}\right]$ being arbitrary constants, real or complex.

The multivariable $\mathbf{H}$-function, introduced and studied by Srivastava and Panda [20] occurring in this paper will be defined in the following contracted form [17, p. 251], eq. (C.1)]

$$
\begin{aligned}
H\left[x_{1}, \ldots, x_{t}\right]=H_{P, Q: P_{1}, Q_{1} ; \ldots ; P_{t}, Q_{t}}^{0, N: M_{1}, N_{1} ; M_{t}, N_{t}}\left[\begin{array}{c|c}
x_{1} & \left(a_{j}: a_{k}^{(1)}, \ldots, a_{k}^{(t)}\right)_{1, P}: \\
\vdots & \begin{array}{l}
\left(b_{j}: \beta_{k}^{(1)}, \ldots, \beta_{k}^{(t)}\right)_{1, Q}: \\
x_{t}
\end{array} \\
\left(c_{k}^{(1)}, \gamma_{k}^{(1)}\right)_{1, P_{1}} ; \ldots ;\left(c_{k}^{(t)}, \gamma_{k}^{(t)}\right)_{1, P_{t}} \\
\left(d_{k}^{(1)}, \delta_{k}^{(1)}\right)_{1, Q_{1}} ; \ldots ;\left(d_{k}^{(t)}, \delta_{k}^{(t)}\right)_{1, Q_{t}}
\end{array}\right] \\
=\frac{1}{(2 \pi \omega)^{t}} \int_{L_{1}} \ldots \int_{L_{t}} \psi\left(\xi_{1}, \ldots, \xi_{t}\right) \phi_{1}\left(\xi_{1}\right) \cdots \phi_{t}\left(\xi_{t}\right) x_{1}^{\xi_{1}}, \ldots, x_{t}^{\xi_{t}} d \xi_{1} \cdots d \xi_{t}
\end{aligned}
$$

where

$$
\begin{aligned}
& \psi\left(\xi_{1}, \ldots, \xi_{t}\right)=\frac{\prod_{k=1}^{N} \Gamma\left(1-a_{k}+\sum_{j=1}^{t} a_{k}^{(j)} \xi_{j}\right)}{\prod_{k=N+1}^{P} \Gamma\left(a_{k}-\sum_{j=1}^{t} a_{k}^{(j)} \xi_{j}\right) \prod_{k=1}^{Q} \Gamma\left(1-b_{k}+\sum_{j=1}^{t} \beta_{k}^{(j)} \xi_{j}\right)} \\
& \phi_{j}\left(\xi_{j}\right)=\frac{\prod_{k=1}^{M_{j}} \Gamma\left(d_{k}^{(j)}-\delta_{k}^{(j)} \xi_{j}\right) \prod_{k=1}^{N_{j}} \Gamma\left(1-c_{k}^{(j)}+\gamma_{k}^{(j)} \xi_{j}\right)}{\prod_{k=M_{j}+1}^{Q_{j}} \Gamma\left(1-d_{k}^{(j)}+\delta_{k}^{(j)} \xi_{j}\right) \prod_{k=N_{j}+1}^{P_{j}} \Gamma\left(c_{k}^{(j)}-\gamma_{k}^{(j)} \xi_{j}\right)} \quad(j=1, \ldots, t)
\end{aligned}
$$

For the convergence and existence conditions of the multivariable $\mathbf{H}$-function, we refer to the book by Srivastava et al. [17, pp. 251-253, eqs. ((C.2)-(C.8))]. It has been assumed throughout the present work that the function satisfies the above cited conditions. 


\section{Eulerian Integrals}

In this section, we shall obtain the following two integral formulas associated with second class of multivariable polynomials and the multivariable $\mathbf{H}$-function as defined in the previous section.

\section{First Integral}

$$
\begin{aligned}
& \int_{a}^{b}(x-a)^{\rho-1}(b-x)^{\sigma-1}(c x+d)^{\eta}(g x+f)^{\omega} S_{V_{1}, \ldots, V_{r}}^{U_{1}, \ldots, U_{r}}\left[y_{1}(x-a)^{\zeta_{1}}(b-x)^{\theta_{1}}(c x+d)^{\lambda_{1}}\right. \\
& \left.(g x+f)^{\delta_{1}}, \ldots, y_{r}(x-a)^{\zeta_{r}}(b-x)^{\theta_{r}}(c x+d)^{\lambda_{r}}(g x+f)^{\delta_{r}}\right] \times H\left[z_{1} \frac{(x-a)^{p_{1}}(b-x)^{q_{1}}}{(c x+d)^{r_{1}}(g x+f)^{s_{1}}},\right. \\
& \left.\ldots, z_{t} \frac{(x-a)^{p_{t}}(b-x)^{q_{t}}}{(c x+d)^{r_{t}}(g x+f)^{s_{t}}}\right] d x=(b-a)^{\rho+\sigma-1}(a c+d)^{\eta}(b g+f)^{\omega} \\
& \sum_{k_{1}=0}^{\left[V_{1} / U_{1}\right]} \cdots \sum_{k_{r}=0}^{\left[V_{r} / U_{r}\right]}\left(-V_{1}\right)_{U_{1} k_{1}} \cdots\left(-V_{r}\right)_{U_{r} k_{r}} A\left(V_{1}, k_{1} ; \ldots ; V_{r}, k_{r}\right) \prod_{i=1}^{r}\left\{\frac{y_{i}^{k_{i}}}{k_{1} !}\right. \\
& \left.\times(b-a)^{\left(\rho_{i}+\theta_{i}\right) k_{i}}(a c+d)^{\lambda_{i} k_{i}}(b g+f)^{\delta_{i} k_{i}}\right\} \times H_{P+4, Q+3: M_{1}, N_{1}, \ldots ; Q_{1} ; \ldots ; M_{t}, N_{t}, Q_{t} ; 0,1,0,1 ; 0,1}^{0, N+1,1} \\
& {\left[I_{1}, \ldots, I_{t}, \frac{(b-a) c}{a c+d}, \frac{-(b-a) g}{b g+f} \mid \begin{array}{l}
A: C ;-;- \\
B: D ;(0,1) ;(0,1)
\end{array}\right]}
\end{aligned}
$$

where

$$
\begin{aligned}
I_{j}= & \frac{z_{j}(b-a)^{p_{j}+q_{j}}}{(a c+d)^{r_{j}}(b g+f)^{s_{j}}} \quad(j=1, \ldots, t) \\
A= & \left(1-\rho-\sum_{i=1}^{r} \zeta_{i} k_{i} ; p_{1}, \ldots, p_{t}, 1,0\right),\left(1-\sigma-\sum_{i=1}^{r} \theta_{i} k_{i} ; q_{1}, \ldots, q_{t}, 0,1\right), \\
& \left(1+\eta+\sum_{i=1}^{r} \lambda_{i} k_{i} ; r_{1}, \ldots, r_{t}, 1,0\right),\left(1+\omega+\sum_{i=1}^{r} \delta_{i} k_{i} ; s_{1}, \ldots, s_{t}, 0,1\right), \\
& \left(a_{k}, a_{k}^{(1)}, \ldots, a_{k}^{(t)}, 0,0\right)_{1, P} \\
B= & \left(1+\eta+\sum_{i=1}^{r} \lambda_{i} k_{i} ; r_{1}, \ldots, r_{t}, 0,0\right),\left(1+\omega+\sum_{i=1}^{r} \delta_{i} k_{i} ; s_{1}, \ldots, s_{t}, 0,0\right), \\
& \left(1-\rho-\sigma-\sum_{i=1}^{r}\left(\zeta_{i}+\theta_{i}\right) k_{i} ; p_{1}+q_{1}, \ldots, p_{t}+q_{t}, 1,1\right),\left(b_{k}, \beta_{k}^{(1)}, \ldots, \beta_{k}^{(t)}, 0,0\right)_{1, Q} \\
C= & \left(c_{k}^{(1)}, \gamma_{k}^{(1)}\right)_{1, P_{1}} ; \ldots ;\left(c_{k}^{(t)}, \gamma_{k}^{(t)}\right)_{1, P_{t}} \\
D= & \left(d_{k}^{(1)}, \delta_{k}^{(1)}\right)_{1, Q_{1}} ; \ldots ;\left(d_{k}^{(t)}, \delta_{k}^{(t)}\right)_{1, Q_{t}}
\end{aligned}
$$

provided that the following conditions are satisfied

(i) $\operatorname{Re}(\rho, \sigma)>0$ 
(ii) $\min \left(p_{j}, q_{j}, r_{j}, s_{j}\right) \geq 0(j=1, \ldots, t)$ (not all zero simultaneously)

(iii) When min $\left(\zeta_{i}, \theta_{i}\right) \geq \mathbf{0}(\boldsymbol{i}=\mathbf{1}, \ldots, \boldsymbol{r})$

$$
\begin{gathered}
\boldsymbol{\operatorname { R e }}[\rho]+\sum_{j=1}^{t} p_{j} \min _{1 \leq k \leq M_{j}} \boldsymbol{\operatorname { R e }}\left(\frac{d_{k}^{(j)}}{\delta_{k}^{(j)}}\right)>0 \\
\boldsymbol{\operatorname { R e }}[\sigma]+\sum_{j=1}^{t} q_{j} \min _{1 \leq k \leq M_{j}} \boldsymbol{\operatorname { R e }}\left(\frac{d_{k}^{(j)}}{\delta_{k}^{(j)}}\right)>0
\end{gathered}
$$

When $\max \left(\zeta_{i}, \theta_{i}\right)<\mathbf{0}(i=1, \ldots, r)$

$$
\begin{gathered}
\boldsymbol{\operatorname { R e }}[\rho]+\sum_{i=1}^{r} \zeta_{i}\left[\frac{V_{i}}{U_{i}}\right]+\sum_{j=1}^{t} p_{j} \min _{1 \leq k \leq M_{j}} \operatorname{Re}\left(\frac{d_{k}^{(j)}}{\delta_{k}^{(j)}}\right)>0 \\
\boldsymbol{\operatorname { R e }}[\sigma]+\sum_{i=1}^{r} \theta_{i}\left[\frac{V_{i}}{U_{i}}\right]+\sum_{j=1}^{t} q_{j} \min _{1 \leq k \leq M_{j}} \boldsymbol{\operatorname { R e }}\left(\frac{d_{k}^{(j)}}{\delta_{k}^{(j)}}\right)>0
\end{gathered}
$$

When $\zeta_{\boldsymbol{i}} \geq \mathbf{0}, \boldsymbol{\theta}_{\boldsymbol{i}}<\mathbf{0},(\boldsymbol{i}=\mathbf{1}, \ldots, \boldsymbol{r}),(\mathbf{I})$ and $(\mathbf{I V})$ are satisfied

When $\boldsymbol{\theta}_{\boldsymbol{i}} \geq \mathbf{0}, \boldsymbol{\zeta}_{\boldsymbol{i}}<\mathbf{0},(\boldsymbol{i}=\mathbf{1}, \ldots, \boldsymbol{r}),(\mathbf{I I})$ and (III) are satisfied (iv) $\max \left\{\left|\frac{(b-a) c}{a c+d}\right|,\left|\frac{(b-a) g}{b g+f}\right|\right\}<1 ; b \neq a$

\section{Second Integral}

$$
\begin{aligned}
& \int_{a}^{b}(x-a)^{\rho-1}(b-x)^{\sigma-1}(c x+d)^{\eta}(g x+f)^{\omega} S_{V_{1}, \ldots, V_{r}}^{U_{1}, \ldots, U_{r}}\left[y_{1}(x-a)^{\zeta_{1}}(b-x)^{\theta_{1}}(c x+d)^{\lambda_{1}}\right. \\
& \left.(g x+f)^{\delta_{1}}, \ldots, y_{r}(x-a)^{\zeta_{r}}(b-x)^{\theta_{r}}(c x+d)^{\lambda_{r}}(g x+f)^{\delta_{r}}\right] \times H^{*}\left[z_{1} \frac{(c x+d)^{r_{1}}(g x+f)^{s_{1}}}{(x-a)^{p_{1}}(b-x)^{q_{1}}},\right. \\
& \left.\ldots, z_{t} \frac{(c x+d)^{r_{t}}(g x+f)^{S_{t}}}{(x-a)^{p_{t}}(b-x)^{q_{t}}}\right] d x=(b-a)^{\rho+\sigma-1}(a c+d)^{\eta}(b g+f)^{\omega} \\
& \times \sum_{k_{1}=0}^{\left[V_{1} / U_{1}\right]}\left[\sum _ { k _ { r } = 0 } ^ { [ V _ { r } / U _ { r } ] } ( - V _ { 1 } ) _ { U _ { 1 } k _ { 1 } } \ldots ( - V _ { r } ) _ { U _ { r } k _ { r } } A ( V _ { 1 } , k _ { 1 } ; \ldots ; V _ { r } , k _ { r } ) \prod _ { j = 1 } ^ { r } \left\{\frac{y_{i}^{k_{i}}}{k_{i} !}(b-a)\left(\zeta_{i}+\theta_{i}\right) k_{i}\right.\right. \\
& \left.\times(a c+d)^{\lambda_{i} k_{i}}(b g+f)^{\delta_{i} k_{i}}\right\} \times H_{Q+4, P+3: Q_{1}, P_{1} ; \ldots ; Q_{t}, P_{t} ; 0,1 ; 0,1}^{0,4: N_{1}, M_{1} ; \ldots ; N_{t}, M_{t} ; 1,0 ; 1,0} \\
& {\left[J_{1}, \ldots, J_{t}, \frac{c(b-a)}{a c+d}, \frac{-g(b-a)}{b g+f} \mid \begin{array}{l}
A^{*}: C^{*} ;-;- \\
B^{*}: D^{*} ;(0,1) ;(0,1)
\end{array}\right]}
\end{aligned}
$$

where

$H^{*}\left[z_{1}, \ldots, z_{t}\right]$ stands for $H\left[z_{1}, \ldots, z_{t}\right]$ with $N=0$.

$$
J_{j}=\frac{1}{z_{j}} \frac{(b-a)^{p_{j}+q_{j}}}{(a c+d)^{r_{j}}(b g+f)^{s_{j}}} \quad(j=1, \ldots, t)
$$




$$
\begin{aligned}
A^{*}= & \left(1-\rho-\sum_{i=1}^{r} \zeta_{i} k_{i} ; p_{1}, \ldots, p_{t}, 1,0\right),\left(1-\sigma-\sum_{i=1}^{r} \theta_{i} k_{i} ; q_{1}, \ldots, q_{t}, 0,1\right) \\
& \left(1+\eta+\sum_{i=1}^{r} \lambda_{i} k_{i} ; r_{1}, \ldots, r_{t}, 1,0\right),\left(1+\omega+\sum_{i=1}^{r} \delta_{i} k_{i} ; s_{1}, \ldots, s_{t}, 0,1\right) \\
& \left(1-b_{k}, \beta_{k}^{(1)}, \ldots, \beta_{k}^{(t)}, 0,0\right)_{1, Q} \\
B^{*}= & \left(1+\eta+\sum_{i=1}^{r} \lambda_{i} k_{i} ; r_{1}, \ldots, r_{t}, 0,0\right),\left(1+\omega+\sum_{i=1}^{r} \delta_{i} k_{i} ; s_{1}, \ldots, s_{t}, 0,0\right) \\
& \left(1-\rho-\sigma-\sum_{i=1}^{r}\left(\zeta_{i}+\theta_{i}\right) k_{i} ; p_{1}+q_{1}, \ldots, p_{t}+q_{t}, 1,1\right),\left(1-a_{k}, a_{k}^{(1)}, \ldots, a_{k}^{(t)}, 0,0\right)_{1, P}(2.1 \\
C^{*}= & \left(1-d_{k}^{(1)}, \delta_{k}^{(1)}\right)_{1, Q_{1}} ; \ldots ;\left(d_{k}^{(t)}, \delta_{k}^{(t)}\right)_{1, Q_{t}} \\
D^{*}= & \left(1-c_{k}^{(1)}, \gamma_{k}^{(1)}\right)_{1, P_{1}} ; \ldots ;\left(c_{k}^{(t)}, \gamma_{k}^{(t)}\right)_{1, P_{t}}
\end{aligned}
$$

provided that the following conditions are satisfied

(i) $\operatorname{Re}(\rho, \sigma)>0$

(ii) $\min \left(p_{j}, q_{j}, r_{j}, s_{j}\right) \geq 0(j=1, \ldots, t)$ (not all zero simultaneously)

(iii) When min $\left(\zeta_{i}, \theta_{i}\right) \geq 0(i=1, \ldots, r)$

$$
\begin{gathered}
\boldsymbol{\operatorname { R e }}[\rho]+\sum_{j=1}^{t} p_{j} \min _{1 \leq k \leq N_{j}} \boldsymbol{\operatorname { R e }}\left(\frac{c_{k}^{(j)}-1}{\gamma_{k}^{(j)}}\right)>0 \\
\boldsymbol{\operatorname { R e }}[\sigma]-\sum_{j=1}^{t} q_{j} \max _{1 \leq k \leq N_{j}} \boldsymbol{\operatorname { R e }}\left(\frac{c_{k}^{(j)}-1}{\gamma_{k}^{(j)}}\right)>0
\end{gathered}
$$

When $\max \left(\zeta_{i}, \theta_{i}\right)<0(i=1, \ldots, r)$

$$
\begin{gathered}
\boldsymbol{\operatorname { R e }}[\rho]+\sum_{i=1}^{r} \zeta_{i}\left[\frac{V_{i}}{U_{i}}\right]+\sum_{j=1}^{t} p_{j} \max _{1 \leq k \leq N_{j}} \operatorname{Re}\left(\frac{c_{k}^{(j)}-1}{\gamma_{k}^{(j)}}\right)>0 \\
\mathbf{R e}[\sigma]+\sum_{i=1}^{r} \theta_{i}\left[\frac{V_{i}}{U_{i}}\right]-\sum_{j=1}^{t} q_{j} \max _{1 \leq k \leq N_{j}} \operatorname{Re}\left(\frac{c_{k}^{(j)}-1}{\gamma_{k}^{(j)}}\right)>0
\end{gathered}
$$

When $\boldsymbol{\zeta}_{\boldsymbol{i}} \geq \mathbf{0}, \boldsymbol{\theta}_{\boldsymbol{i}}<\mathbf{0},(\boldsymbol{i}=\mathbf{1}, \ldots, \boldsymbol{r}),(\mathbf{V})$ and (VIII) are satisfied

When $\boldsymbol{\theta}_{\boldsymbol{i}} \geq \mathbf{0}, \boldsymbol{\zeta}_{\boldsymbol{i}}<\mathbf{0},(\boldsymbol{i}=\mathbf{1}, \ldots, \boldsymbol{r}),(\mathrm{VI})$ and (VII) are satisfied (iv) $\max \left\{\left|\frac{(b-a) \bar{c}}{a c+d}\right|,\left|\frac{(b-a) g}{b g+f}\right|\right\}<1 ; b \neq a$

Proof. To establish the integral given by (2.1), we first express the multivariable $\mathbf{H}$ function and the second class of multivarible polynominal occurring in the left hand side of (2.1) with the help of (1.3) and (1.2) respectively, then collect the powers of $(c x+d)$ 
and $(g x+f)$ and apply the following binomial expansions for $x \in[a, b]$

$$
\begin{aligned}
& (c x+d)^{\eta}=(a c+d)^{\eta} \sum_{l_{1}=0}^{\infty} \frac{(-\eta) l_{1}}{l_{1} !}\left\{\frac{-(x-a) c}{a c+d}\right\}^{l_{1}},|(x-a) c|<|a c+d| \\
& (g x+f)^{\omega}=(b g+f)^{\omega} \sum_{l_{2}=0}^{\infty} \frac{(-\omega) l_{2}}{l_{2}}\left\{\frac{+(b-x) g}{b g+f}\right\}^{l_{2}},|(b-x) g|<|b g+f|
\end{aligned}
$$

with $\eta$ and $\omega$ replaced by $\eta+\sum_{i=1}^{r} \lambda_{i} k_{i}-\sum_{j=1}^{t} r_{j} \xi_{j}$ and $\omega+\sum_{i=1}^{r} \delta_{i} k_{i}-\sum_{j=1}^{t} s_{j} \xi_{j}$ respectively. Here $\xi_{1}, \ldots, \xi_{t}$ denote the variables of the aforementioned Mellin Barnes contour integral of $\mathbf{H}$-function. Finally, making use of well known Eulerian integral

$$
\int_{a}^{b}(x-a)^{\rho-1}(b-x)^{\sigma-1} d x=(b-a)^{\rho+\sigma-1} \mathbf{B}(\rho, \sigma) ; \operatorname{Re}(\rho)>0, \boldsymbol{R e}(\sigma)>0 ; b \neq a
$$

and iterpreting the resulting Mellin Barnes contour integral as an $\mathbf{H}$-function of $(t+2)$ variables, we arrive at the right hand side of (2.1) after a little simplification. To prove the second integral given by (2.7), we follow the same lines as in the proof of (2.1) except the following changes. Here, we apply the binomial expansions (2.13) and (2.14) with $\eta$ and $\omega$ replaced by $\eta+\sum_{i=1}^{r} \lambda_{i} k_{i}+\sum_{j=1}^{t} r_{j} \xi_{j}$ and $\omega+\sum_{i=1}^{r} \delta_{i} k_{i}+\sum_{j=1}^{t} s_{j} \xi_{j}$ respectively and then set $\xi_{j}=-\zeta_{j}(j=1, \ldots, t)$ to interpret the resulting Mellin Barnes contour integral as an $\mathbf{H}$-function of $(t+2)$ variables and arrive at right hand side of (2.7).

Two more integral formulas, involving the product of first class of multivariable polynomials $S_{V}^{U_{1}, \ldots, U_{r}}\left[x_{1}, \ldots, x_{r}\right]$ and the multivariable $\mathbf{H}$-function, can also be obtained similarly. However, we omit the details here.

\section{Special Cases}

Each of our integral formulas (2.1) and (2.7) possesses manifold generality. The multivariable $\mathbf{H}$-function occurring in these results can be suitably specialized to yield a wide variety of special functions (or product of such functions) of one or more variables [17, pp. 18-19, 88-93, 253-254]. Again by suitably specializing the coefficient of the general class of multivariable polynomials it can be reduced to other multivariable hypergeometric polynomials and classical orthogonal polynomials of one or more variables.

Thus our results and their several variations (obtained by letting any desired number of exponents $\left\{\zeta_{i}, \theta_{i}, \lambda_{i}, \delta_{i}, p_{j}, q_{j}, r_{j}, s_{j}(i=1, \ldots, r ; j=1, \ldots, t)\right\}$ tend to zero in such a manner that each side of resulting equation has a meaning) would yield the corresponding results involving a large number of simpler functions and polynomials.

To illustrate we now obtain some new special cases of our result (2.1) and also give references to several known results which follow as special cases of integral formulas (2.1) and (2.7).

(i) On taking $A\left(V_{1}, k_{1} ; \ldots ; V_{r}, k_{r}\right)=A_{1}\left(V_{1}, k_{1}\right) \cdots A_{r}\left(V_{r}, k_{r}\right)$ in $(2.1)$, the multivariable polynomial $S_{V_{1}, \ldots, V_{r}}^{U_{1}, \ldots, U_{r}}\left[x_{1}, \ldots, x_{r}\right]$ reduces to the product of $\mathbf{r}$ well known general class 
of polynomial $S_{V}^{U}[x]$ introduced by Srivastava [[13, p.1, eq. (1)]; for more details see [21]] and the result assumes the following form

\section{Third Integral}

$$
\begin{aligned}
& \int_{a}^{b}(x-a)^{\rho-1}(b-x)^{\sigma-1}(c x+d)^{\eta}(g x+f)^{\omega} \prod_{i=1}^{r}\left[S _ { V _ { i } } ^ { U _ { i } } \left[y_{i}(x-a)^{\zeta_{i}}(b-x)^{\theta_{i}}(c x+d)^{\lambda_{i}}\right.\right. \\
& \left.\left.(g x+f)^{\delta_{i}}\right]\right] H\left[z_{1} \frac{(x-a)^{p_{1}}(b-x)^{q_{1}}}{(c x+d)^{r_{1}}(g x+f)^{s_{1}}}, \ldots, z_{t} \frac{(x-a)^{p_{t}}(b-x)^{q_{t}}}{(c x+d)^{r_{t}}(g x+f)^{s_{t}}}\right] d x \\
= & (b-a)^{\rho+\sigma-1}(a c+d)^{\eta}(b g+f)^{\omega} \prod_{i=1}^{r}\left\{\sum_{k_{i}=0}^{\left[V_{i} / U_{i}\right]}\left(-V_{i}\right)_{U_{i} k_{i}} A_{i}\left(V_{i}, k_{i}\right) \frac{y_{i}^{k_{i}}}{k_{i} !}\right. \\
& \left.\times(b-a)^{\left(\zeta_{i}+\theta_{i}\right) k_{i}}(a c+d)^{\lambda_{i} k_{i}}(b g+f)^{\delta_{i} k_{i}}\right\} H\left[I_{1}, \ldots, I_{t}, \frac{(b-a) c}{a c+d}, \frac{-(b-a) g}{b g+f}\right]
\end{aligned}
$$

where the $\mathbf{H}$-function occurring in the right hand side of (3.1) is same as in right hand side of (2.1) and the conditions mentioned with (2.1) are assumed to be satisfied.

(ii) On taking

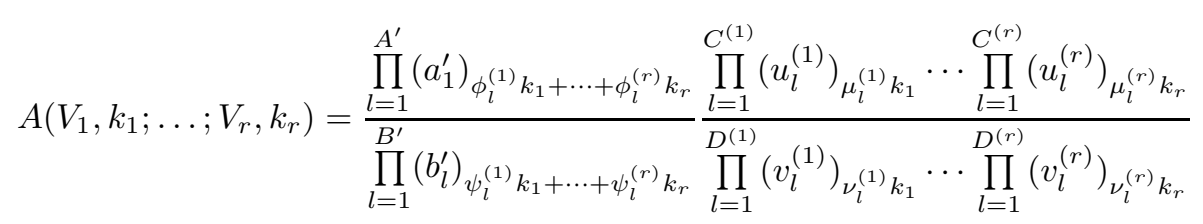

in (2.1), $S_{V_{1}, \ldots, V_{r}}^{U_{1}, \ldots, U_{r}}\left[x_{1}, \ldots, x_{r}\right]$ reduces to the polynomial form of the generalized Lauricella function of Srivastava and Daoust [15, p. 454] as follows

$$
\begin{aligned}
& S_{V_{1}, \ldots, V_{r}}^{U_{1}, \ldots U_{r}}\left[x_{1}, \ldots, x_{r}\right]=F_{B^{\prime}: D^{(1)}, \ldots, D^{(r)}}^{A^{\prime}: C^{(1)}+1, \ldots, C^{(r)}+1} \\
& {\left[\begin{array}{l}
\left(a_{l}^{\prime}: \phi_{l}^{(1)}, \ldots, \phi_{l}^{(r)}\right)_{1, A^{\prime}},\left(u_{l}^{(1)}, \mu_{l}^{(1)}\right)_{1, C^{(1)}},\left(-V_{1}, U_{1}\right), \ldots,\left(u_{l}^{(r)}, \mu_{l}^{(r)}\right)_{1, C^{(r)},},\left(-V_{r}, U_{r}\right) \\
\left(b_{l}^{\prime}: \psi_{l}^{(1)}, \ldots, \psi_{l}^{(r)}\right)_{1, B^{\prime}},\left(v_{l}^{(1)}, \nu_{l}^{(1)}\right)_{1, D^{(1)}}, \ldots,\left(v_{l}^{(r)}, \nu_{l}^{(r)}\right)_{1, D^{(r)}}
\end{array}\right]}
\end{aligned}
$$

and we easily arrive at an Eulerian integral involving product of general Lauricella function and the multivarible $\mathbf{H}$-function. We however, omit the details of the result due to lack of space.

(iii) On taking $A\left(V_{1}, k_{1} ; \ldots ; V_{k}, k_{r}\right)=\frac{(a+1)_{V_{1}+\cdots+V_{r}}}{V_{1} ! \cdots V_{r} !} \times \frac{1}{(\alpha+1)_{\mu k_{1}+\cdots+\mu k_{r}}}(\mu$ being arbitrary) and $U_{i}=1(i=1, \ldots, r)$ in $(2.1), S_{V_{1}, \ldots, V_{r}}^{U_{1}, \ldots, U_{r}}\left[x_{1}, \ldots, x_{r}\right]$ reduces to the polynomials $L_{V_{1}, \ldots, V_{r}}^{\alpha, \mu}\left[x_{1}, \ldots, x_{r}\right]$ which are an extension, due to Chak [3, p.14, eq.(1.1)], of the generalized Laguerre polynomials in several variables studied by Erdélyi [4] and the result (2.1) assumes the following form 


\section{Fourth Integral}

$$
\begin{aligned}
& \int_{a}^{b}(x-a)^{\rho-1}(b-x)^{\sigma-1}(c x+d)^{\eta}(g x+f)^{\omega} L_{V_{1}, \ldots, V_{r}}^{\alpha, \mu}\left[y_{1}(x-a)^{\zeta_{1}}(b-x)^{\theta_{1}}(c x+d)^{\lambda_{1}}\right. \\
& \left.(g x+f)^{\delta_{1}}, \ldots, y_{r}(x-a)^{\zeta_{r}}(b-x)^{\theta_{r}}(c x+d)^{\lambda_{r}}(g x+f)^{\delta_{r}}\right] H\left[z_{1} \frac{(x-a)^{p_{1}}(b-x)^{q_{1}}}{(c x+d)^{r_{1}}(g x+f)^{s_{1}}}\right. \\
& \left.\ldots, z_{t} \frac{(x-a)^{p_{t}}(b-x)^{q_{t}}}{(c x+d)^{r_{t}}(g x+f)^{s_{t}}}\right] d x=(b-a)^{\rho+\sigma-1}(a c+d)^{\eta}(b g+f)^{\omega} \frac{(a+1)_{V_{1}+\cdots+V_{r}}}{V_{1} ! \cdots V_{r} !} \\
& \sum_{k_{1}=0}^{V_{1}} \ldots \sum_{k_{r}=0}^{V_{r}} \frac{\left(-V_{1}\right)_{k_{1}} \cdots\left(-V_{r}\right)_{k_{r}}}{(\alpha+1)_{\mu k_{1}+\cdots+\mu k_{r}}} \prod_{i=1}^{r}\left\{\frac{y_{i}^{k_{i}}}{k_{i} !}(b-a)^{\left(\zeta_{i}+\theta_{i}\right) k_{i}}(a c+d)^{\lambda_{i} k_{i}}(b g+f)^{\delta_{i} k_{i}}\right\} \\
& \times H\left[I_{1}, \ldots, I_{t}, \frac{(b-a) c}{a c+d}, \frac{-(b-a) g}{b g+f}\right]
\end{aligned}
$$

where $\mathbf{H}$-function occurring in the right hand side of (3.4) is same as in right hand side of (2.1) and the conditions of validity of (3.4) can easily be obtained from those stated with (2.1).

(iv) If in integral formulas (2.1) and (2.7) we take $V_{i}=0(i=1, \ldots, r)$, reduce the multivariable polynomials to unity and further take $p_{j}=q_{j}=0(j=1, \ldots, t)$, we arrive at the integral evaluated recently by Srivastava and Hussain [18, p. 79, eqs. (2.5), (2.6)] after a little simplification.

In the results thus obtained if we further take $\omega=0, s_{j}=0(j=1, \ldots, t)$ and reduce the multivarible $\mathbf{H}$-function to product of ' $t$ ' Fox's $\mathbf{H}$-function (by taking $N=P=Q=$ 0 ), then on taking $t=1$, we arrive at the integrals evaluated recently by Saxena and Nishimoto [11, p. 69, eq. (4.1)].

(v) On taking $V_{i}=0(i=1, \ldots, r)$ in $(2.1)$ and reducing the general class of multivariable polynomials to unity. Further, on taking $\omega=0, s_{j}=0(j=1, \ldots, t)$ and reducing the multivarible $\mathbf{H}$-function to product of ' $t$ ' Fox's $\mathbf{H}$-function (by taking $N=P=Q=0)$, then on taking $t=1$, we arrive at the integral evaluated recently by Saxena and Saigo [12, p. 37, eq. (2.1)]

(vi) If we take $r=1$ in (2.1), the general class of multivariable polynomials reduces to $S_{V}^{U}[x]$, and further on taking $\theta=1, \zeta=\lambda=\delta=0, r_{j}=s_{j}=0(j=1, \ldots, t)$ therein, we easily arrive at an integral obtained earlier by Srivastava and Singh [21, p. 166, eq. $(2.2)]$

(vii) Next on reducing the $\mathbf{H}$-function occurring in the left hand side of (2.1) to unity, and setting $\eta=-\rho-\sigma, c=\lambda-\mu, d=(1+\mu) b-(1+\lambda) a$ and $\lambda_{i}=\delta_{i}=0, \zeta_{i}=1$, $\theta_{i}=-1(i=1, \ldots, r)$ therein, we arrive at the result recently obtained by Saigo and Raina $[9$, eq. $(2.1)]$ 


\section{Applications}

Each of the Eulerian integrals obtained in previous sections can be expressed as fractional integrals involving the operator ${ }_{a} D_{z}^{-\nu}$ defined by [see [7], [10]]

$$
{ }_{a} D_{z}^{-\nu}[f(z)]=\frac{1}{\Gamma(\nu)} \int_{a}^{z}(z-t)^{\nu-1} f(t) d t ; \quad \operatorname{Re}(\nu)>0
$$

when $a=0$, the fractional integral operator (4.1) corresponds to the classical RiemannLiouville fractional integral of order $\nu$. Moreover, when $a \rightarrow \infty$, equation may be identified with the definition of Weyl fractional integral of order $\nu$. Thus for example, for $b=z, \theta_{i}=0=q_{j}(i=1, \ldots, r ; j=1, \ldots, t)$, we express below the integral $(2.1)$ as fractional integral formula which is valid under the conditions easily obtained from those state with (2.1)

$$
\begin{aligned}
& { }_{a} D_{z}^{-\sigma}\left\{(z-a)^{\rho-1}(c z+d)^{\eta}(g z+f)^{\omega}\right. \\
& \times S_{V_{1}, \ldots, V_{r}}^{U_{1}, \ldots, U_{r}}\left[y_{1}(z-a)^{\rho-1}(c z+d)^{\lambda_{1}}(g z+f)^{\delta_{1}}, \ldots, y_{r}(z-a)^{\rho_{r}}(c+d)^{\lambda_{r}}(g z+f)^{\delta_{r}}\right] \\
& \left.\times H\left[z_{1} \frac{(z-a)^{p_{1}}}{(c z+d)^{r_{1}}(g z+f)^{s_{1}}}, \ldots, z_{t} \frac{(z-a)^{p_{t}}}{(c z+d)^{r_{t}}(g z+f)^{s_{t}}}\right]\right\}=\frac{1}{\Gamma(\sigma)}(z-a)^{p+\sigma-1}(a c+d)^{\eta} \\
& \cdot(g z+f)^{\omega} \sum_{k_{1}=0}^{\left[V_{1} / U_{1}\right]} \cdots \sum_{k_{r}=0}^{\left[V_{r} / U_{r}\right]}\left(-V_{1}\right)_{U_{1} k_{1}} \cdots\left(-V_{r}\right)_{U_{r} k_{r}} \times A\left(V_{1}, k_{1} ; \ldots ; V_{r}, k_{r}\right) \\
& \prod_{i=1}^{r}\left\{\frac{y_{i}^{k_{i}}}{k_{i} !}(b-a)^{\rho_{i} k_{i}}(a c+d)^{\lambda_{i} k_{i}}(g z+f)^{\delta_{i} k_{i}}\right\} \times H_{P+3, Q+3: M_{1}, N_{1} ; \ldots ; Q_{1} ; Q_{t} ; \ldots ; P_{t}, Q_{t} ; Q_{t} ; 0 ; 1,1,1 ; 1,1}^{0, N+3 ;}, \\
& {\left[K_{i}, \ldots, K_{t}, \frac{c(z-a)}{a c+d}, \frac{-g(z-a)}{g z+f} \mid \begin{array}{l}
A^{* *}: C ;-;(1-\sigma, 1) \\
B^{* *}: D ;(0,1) ;(0,1)
\end{array}\right]}
\end{aligned}
$$

where $K_{j}$ stands for $I_{j}$ as given by $(2.2)$ with $q_{j}=0(j=1, \ldots, t), C$ and $D$ are given by (2.5) and (2.6) respectively.

$$
\begin{aligned}
A^{* *}= & \left(1+\eta+\sum_{i=1}^{r} \lambda_{i} k_{i} ; r_{1}, \ldots, r_{t}, 1,0\right),\left(1+\omega+\sum_{i=1}^{r} \delta_{i} k_{i} ; s_{1}, \ldots, s_{t}, 0,1\right), \\
& \left(1-\rho-\sum_{i=1}^{r} \zeta_{i} k_{i} ; p_{1}, \ldots, p_{t}, 1,0\right),\left(a_{k} ; a_{k}^{(1)}, \ldots, a_{k}^{(t)}, 0,1\right)_{1, P} \\
B^{* *}= & \left(1+\eta+\sum_{i=1}^{r} \lambda_{i} k_{i} ; r_{1}, \ldots, r_{t}, 0,0\right),\left(1+\omega+\sum_{i=1}^{r} \delta_{i} k_{i} ; s_{1}, \ldots, s_{t}, 0,0\right), \\
& \left(1-\rho-\sigma-\sum_{i=1}^{r} \zeta_{i} k_{i} ; p_{1}, \ldots, p_{t}, 1,1\right),\left(b_{k} ; \beta_{k}^{(1)}, \ldots, \beta_{k}^{(t)}, 0,0\right)_{1, Q}
\end{aligned}
$$

\section{References}


[1] L. Carlitz, Bilinear generating functions for Laguerre and Lauricella polynomials in several variables, Rend. Sem. Mat. Univ. Padova, 43(1970b), 269-276.

[2] L. Carlitz and H. M. Srivastava, Some hypergeometric polynomials associated with the Lauricella function $F_{D}$ of several variables, I and II, Mat. Vesnik, 13, 41-47 and 143-152.

[3] A. M. Chak, Some generalizations of Laguerre polynomials, I and II, Mat. Vesnik, 7(1970), $7-18$.

[4] A. Erdélyi, Beitrag zur Theorie der Konflventen hypergeometrischen Funktionen von mehreren veranderlichen, S. -B. Akad. Wiss. Wien Abt. IIa Math. -Natur. K1., 146 (1937a), 431-467.

[5] M. Garg and M. K. Gupta, On a unified finite integral, Jñánábha, 27(1997), to appear.

[6] K. C. Gupta and R. C. Soni, On unified integrals, J. Indian Math. Soc. (N.S.), 55(1990), 49-58.

[7] K. S. Miller and B. Ross, An introduction to the Fractional Calculus and Fractional Differential Equations, John Wiley and Sons, New York, 1993.

[8] R. K. Raina and H. M. Srivastava, Evaluation of certain class of Eulerian integrals, J. Phys. A: Math. Gen., 26(1993), 691-696.

[9] M. Saigo and R. K. Raina, Evaluation of certain integrals in terms of generalized Kampé de Fériet function and Lauricella function $F_{D}^{(n)}$, Tamkang, J. Math., 26(1995), 41-47.

[10] S. G. Samko, A. A. Kilbas and O. I. Marichev, Fractional Integrals and Derivatives, Theory and Applications, Gordon and Breach, New York- Philadelphia -London -Paris -Montreux -Tornoto -Melbourne, 1993.

[11] R. K. Saxena and K. Nishimoto, Fractional integral formula for the H-function, J. Fractional Calculus, 6(1994), 64-75.

[12] R. K. Saxena and M. Saigo, Fractional integral formula for the H-function, II, J. Fractional Calculus, 13(1998), 37-41.

[13] H. M. Srivastava, A contour integral involving Fox's H-function, indian J. Math., 14(1972), $1-6$.

[14] H. M. Srivastava, A multilinear generating function for the Konhauser sets of biorthogonal polynomials suggested by the Laguerre polynomials, Pacific J. Math., 117(1985), 183-191.

[15] H. M. Srivastava and M. C. Daoust, Certain generalized Neumann expansions associated with the Kampé de Fériet function, Nederl. Akad. Wetensch. Proc. Ser. A $72=$ Indag. Math., 31, 449-457.

[16] H. M. Srivastava and M. Garg, Some integrals involving a general class of polynomials and the multivariable H-function, Rev. Roumaine Phys., 32(1987), 685-692.

[17] H. M. Srivastava, K. C. Gupta and S. P. Goyal, The H-Functions of One and Two Variables with Applications, South Asian Publishers, New Delhi and Madras, 1982.

[18] H. M. Srivastava and M. A. Hussain, Fractional integration of the H-function of several variables, Comput. Math. Appl., 30(1995), 73-85.

[19] H. M. Srivastava and H. L. Manocha, A Treatise on Generating Functions, Wiley/Halste, New York, 1984.

[20] H. M. Srivastava and R. Panda, Some bilateral generating functions for a class of generalized hypergeometric polynomials, J. Reine Angew. Math., 283/284(1976), 256-274.

[21] H. M. Srivastava and N. P. Singh, The integration of certain products of the multivariable H-function with a general class of polynomials, Rend. Circ. Mat. Palermo, 32(1983), 157-187.

Department of Mathematics, University of Rajasthan, Jaipur - 302004, Rajasthan, India. 